\title{
Implementación de sistema de recolección de desechos reciclables en la Ciudad Universitaria de San Marcos
}

\section{RESUMEN}

El presente trabajo se enfoca en la necesidad de tratar los residuos urbanos dentro del campus universitario de la UNMSM. En la primera etapa se realizó un diagnóstico del procedimiento de acopio de residuos y su respectiva clasificación y cuantificación. De las alternativas evaluadas para la mejora del sistema de acopio; se determinó que la instalación de una planta de tratamiento de residuos es la más apropiada debido que generara beneficios en el ámbito de salubridad, académico y económico del campus universitario.

Palabras clave: gestión de residuos sólidos, reciclar, planta de tratamiento de residuos

IMPLEMENTING COLLECTION SYSTEM RECYCLABLES IN THE UNIVERSITY CITY of SAN Marcos

\section{ABSTRACT}

This paper focuses on the need to treat urban waste inside the San Marcos university campus In the first stage, a diagnostic procedure for the collection of waste and their respective classification and quantification was performed. Of the alternatives evaluated for improving the collection system, it was determined that the installation of a waste treatment plant is the most appropriate because it will generate benefits in the field of health, academic and economic campus.

Keywords: solid waste management, recycling, waste treatment plant

\section{INTRODUCCIÓN}

Ante la perspectiva de agotamiento de los recursos naturales y como una medida eficaz de eliminar los desechos generados por las diversas actividades que realiza el ser humano, surge el reciclaje. Por ejemplo, por cada tonelada de papel que se recicla, se disminuye la tala de por lo menos 16 árboles que tardan 20 años en crecer. Entre otros beneficios de la implementación del reciclaje en nuestra sociedad se puede mencionar: ahorro de energía, preservar el medioambiente, generar nuevas alternativas de empleo, creación de cultura ambiental y beneficios económicos.

Diversos entes públicos y privados realizan un manejo de sus residuos sólidos con el fin de contribuir con el medio ambiente, tales como la Municipalidad de Surco, el Hospital Rebagliati y la Universidad Privada Ricardo Palma, entre otros.

En lo que respecta a la Universidad Nacional Mayor de San Marcos, el tratamiento de los desechos está dado básicamente por el acopio de los desechos de las facultades, sin previa segregación, en contenedores ubicados dentro de la ciudad universitaria y su posterior traslado hacia rellenos sanitarios, contribuyendo a la contaminación ambiental por la generación de gases de efecto invernadero, lo cual expresa una falta de cultura ambiental y de compromiso social. Esta situación es preocupante si se tiene en consideración que es la universidad pública con mayor número de estudiantes de pregrado, con una tasa de crecimiento de $2.1 \%$, según el censo universitario 2010.

El presente estudio tiene por objetivo la implementacióndeun adecuado sistema de manipulación de desechos reciclables que se generan en los diversos ambientes de la ciudad universitaria cumpliendo los estándares de ecoeficiencia y creando una cultura de reciclaje en la comunidad universitaria de tal forma que pueda servir de referencia para otras instituciones, centros públicos o privados.

Este proyecto además de contribuir con el medio ambiente permitirá generar nuevos puestos de trabajo dentro de la planta de tratamiento de residuos sólidos, así como poner a

\footnotetext{
Bachiller en Ingeniería Industrial, Profesor Principal de la FII-UNMSM. E-mail:jvergiuc@yahoo.com

** Bachiller en Ingeniería Industrial, Profesor Asociado de la FII-UNMSM. E-mail:orojasla@hotmail.com

*** Bachiller en Ingeniería Industrial, Profesor Asociado de la FII-UNMSM. E-mail:javiermejiae@hotmail.com
} 
disposición de los estudiantes un modelo de planta industrial auto financiada.

Según el Acuerdo Nacional Política de Estado $\mathrm{N}^{\circ} 19$ del desarrollo sostenible y gestión ambiental, suscrito el 22 de julio de 2002. Indica el compromiso de integrar la política nacional ambiental con las políticas económicas, sociales, culturales y de ordenamiento territorial,para contribuir a superar la pobreza y lograr el desarrollo sostenible del Perú. Nos comprometemos también a institucionalizar la gestión ambiental, pública y privada, para proteger la diversidad biológica, facilitar el aprovechamiento sostenible de los recursos naturales, asegurarla protección ambiental y promover centros poblados y ciudades sostenibles; lo cual ayudará a mejorar la calidad de vida, especialmente de la población más vulnerable del país.

\section{Con ese objetivo el Estado:}

- Fortalecerá la institucionalidad de la gestión ambiental optimizando la coordinación entre la sociedad civil, la autoridad ambiental nacional, las sectoriales y los niveles de gestión descentralizada, en el marco de un sistema nacional de gestión ambiental;

- Promoverá la participación responsable e informada del sector privado y de la sociedad civil en la toma de decisiones ambientales y en la vigilancia de su cumplimiento, y fomentará una mayor conciencia ambiental;

- Promoverá el ordenamiento urbano, así como el manejo integrado de residuos urbanos e industriales que estimule su reducción, reuso y reciclaje;

- Fortalecerá la educación y la investigación ambiental;

Según el Compendio de la Legislación Ambiental Peruana, volumen V, Calidad Ambiental:

Se puede defender el concepto "calidad ambiental" como el conjunto de características del ambiente, en función a la disponibilidad y facilidad de acceso a los recursos naturales y a la ausencia o presencia de agentes nocivos. Todo esto necesario para el mantenimiento y crecimiento de la calidad de vida de los seres humanos.

Asociados a este concepto, se encuentran los términos "estándar de calidad ambiental" y "límite máximo permisible", instrumentos de gestión ambiental que buscan regular y proteger la salud pública y la calidad ambiental, permitiéndole a la autoridad ambiental desarrollar acciones de control, seguimiento y fiscalización de los efectos causados por las actividades humanas. Un
Estándar de Calidad Ambiental (ECA) es la medida que establece el nivel de contracción o del grado de elementos, sustancias o parámetros físicos, químicos o biológicos, presentes en el aire, agua o suelo, en su condición de cuerpo receptor, que no representa significativo para la salud de las personas ni al ambiente.

\section{MARCO TEÓRICO}

\subsection{Bases Teóricas}

\subsubsection{Residuos Sólidos Orgánicos}

Según Gladys Jaramillo Henao, son aquellos residuos que provienen de restos de productos de origen orgánico, la mayoría de ellos son biodegradables (se descomponen naturalmente). Se pueden desintegrar o degradar rápidamente, transformándose en otro tipo de materia orgánica. Ejemplo: los restos de comida, frutas y verduras, carne, huevos, etcétera, o pueden tener un tiempo de degradación más lento, como el cartón y el papel. Se exceptúa de estas propiedades al plástico, porque a pesar de tener su origen en un compuesto orgánico, posee una estructura molecular más complicada.

\subsubsection{Reciclaje}

Reciclaje [3] es un término empleado de manera general para describir el proceso de utilización de elementos o partes de un artículo que todavía pueden ser usadas a pesar de pertenecer a algo que ya llegó al final de su vida útil. Reciclar es la acción de volver a introducir en el ciclo de producción y consumo, productos y materiales obtenidos de residuos, también se refiere al conjunto de actividades que pretenden reutilizar partes de artículos que en su conjunto han llegado al término de su vida útil, pero que admiten un uso adicional para alguno de sus componentes o elementos.

En una visión ecológica del mundo, el reciclaje es la tercera y última medida en el objetivo de la disminución de residuos; el primero sería la reducción del consumo, y el segundo la reutilización.

\subsubsection{Regla de las tres erres de la Ecología (3R)}

Es una proposición sobre costumbres de consumo, difundida por la organización "Greenpeace", que pretende desarrollar hábitos de consumo responsables.

En la Cumbre del G8 en junio de 2004, el Primer Ministro del Japón, KoizumiJunichiro, presentó la Iniciativa tres erres que busca construir una sociedad orientada hacia el reciclaje. 
Figura 1. Simbología de lasTres Erres.

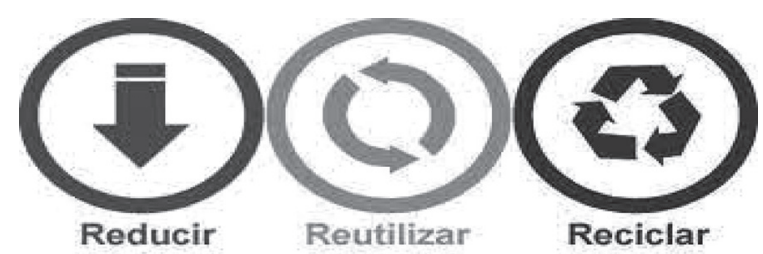

Fuente: Leunam_mr@live.com.mx

Es una regla para cuidar el medio ambiente, específicamente para reducir el volumen de residuos o basura generada, tiene tres pasos: reducir, reutilizar y reciclar.

\subsubsection{Los beneficios de reciclar}

[1] Cada vez que se recicla un envase, se evita llenar los vertederos y la extracción de nuevas materias primas, además se reduce el consumo energético y la emisión de gases contaminantes, causantes del cambio climático. Además, hay más ventajas, con los materiales reciclados se pueden hacer nuevos productos con resultados muy originales y a bajo costo.

\subsection{Marco Conceptual}

Para una mejor comprensión del presente artículo se ha visto conveniente definir los siguientes términos [2]:

- Almacenamiento: operación de acumulación temporal de residuos en condiciones técnicas, como parte del sistema de manejo, hasta su disposición final.

- Contenedor: caja o recipiente fijo o móvil, en el que los residuos se depositan para su almacenamiento o transporte.

- Disposición final: procesos u operaciones para tratar o disponer en un lugar los residuos sólidos, como última etapa de su manejo, en forma permanente, sanitaria y ambientalmente segura.

- Ecosistema: es un complejo dinámico de comunidades vegetales, animales y microorganismos y su medio no viviente que interactúan como una unidad funcional.

- Eficacia: es la consecución de objetivos; logro de los efectos deseados.

- Eficiencia: es el logro de los fines con la menor cantidad de recursos, logro de objetivos al menor costo.
- Reaprovechar: volver a obtener un beneficio del bien, artículo, elemento o parte del mismo que constituye residuo sólido. Se reconoce como técnica de reaprovechamiento el reciclaje, recuperación o reutilización.

- Recolección: acción de recoger los residuos para transferirlos mediante un medio de locomoción apropiado y luego continuar su posterior manejo, en forma sanitaria, segura y ambientalmente adecuada.

- Reducir: minimizar la producción de residuos, adoptando hábitos de "no despilfarro", incrementando el uso de envases retornables, disminuyendo la utilización de plásticos.

- Residuo orgánico: se refiere a los residuos biodegradables o sujetos a descomposición.

- Reutilizar: consiste en no eliminar aquello que ya se considera "inservible", pues todavía puede tener una utilización posterior. Hay objetos que tienen usos alternativos una vez que han sido utilizados.

- Segregación: acción de agrupar determinados componentes o elementos físicos de los residuos sólidos, para ser manejados en forma especial.

\section{DIAGNÓSTICO DEL TRATAMIENTO DE RESI- DUOS SÓLIDOS EN LA UNMSM}

Para el análisis del diagnóstico respecto al reciclaje, se realizó entrevistas y encuestas:

- Personal de mantenimiento y servicio de las facultades

- Personal de mantenimiento y servicio de las oficinas de la administración central.

- Estudiante universitarios,

- Se obtuvieron los siguientes resultados:

\subsection{Conocimiento de la Comunidad Universitaria acerca del proceso del reciclaje}

Para esta investigación se realizaron encuestas a los jefes del personal de limpieza de las diversas facultades, encontrándose lo siguiente:

En ninguna de las facultades encuestadas se ha brindado charla informativa acerca del reciclaje, no se reciben capacitaciones sobre riesgos sanitarios y no cuentan con la indumentaria necesaria para su protección.

En la Figura 2 se observa que solo el $25 \%$ de las facultades encuestadas cuentan con tachos específicos para cada residuo; sin embargo éstos 
no se respetan, utilizándose finalmente como cualquier tacho.

Ante la pregunta sobre los aspectos que dificultan la clasificación de los residuos, tal como se muestra

Figura 2. Porcentaje de facultades con contenedores para separar los residuos.

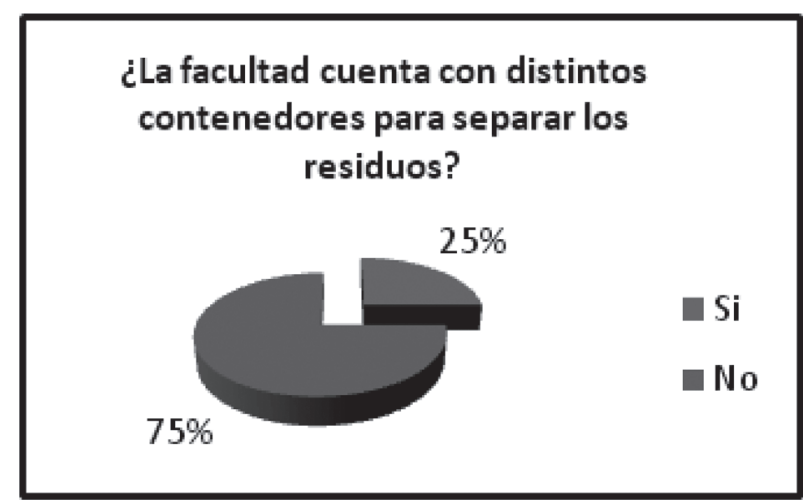

Fuente: Elaboración propia. en la Figura 3, el $50 \%$ indica que se debe al desconocimiento de la forma de hacerlo, el $43 \%$ indica a la falta de cooperación de los alumnos y el personal en general.

Figura 3. Porcentaje de aspectos que dificultan la clasificación de residuos.

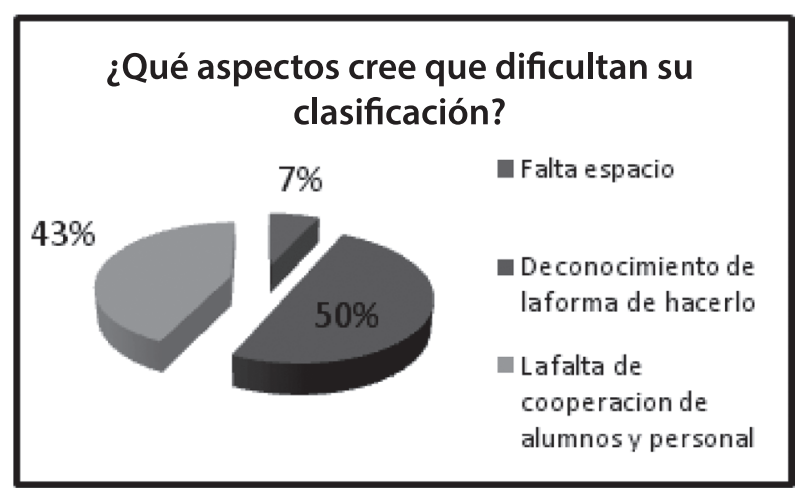

Fuente: Elaboración propia.

Al realizar preguntas referentes a una futura participación en un programa de reciclaje, la comunidad universitaria está dispuesta a apoyar y participar de un programa de reciclaje, representando el $77 \%$.

Figura 4. Porcentaje de facultades que apoyarían un programa de reciclaje.

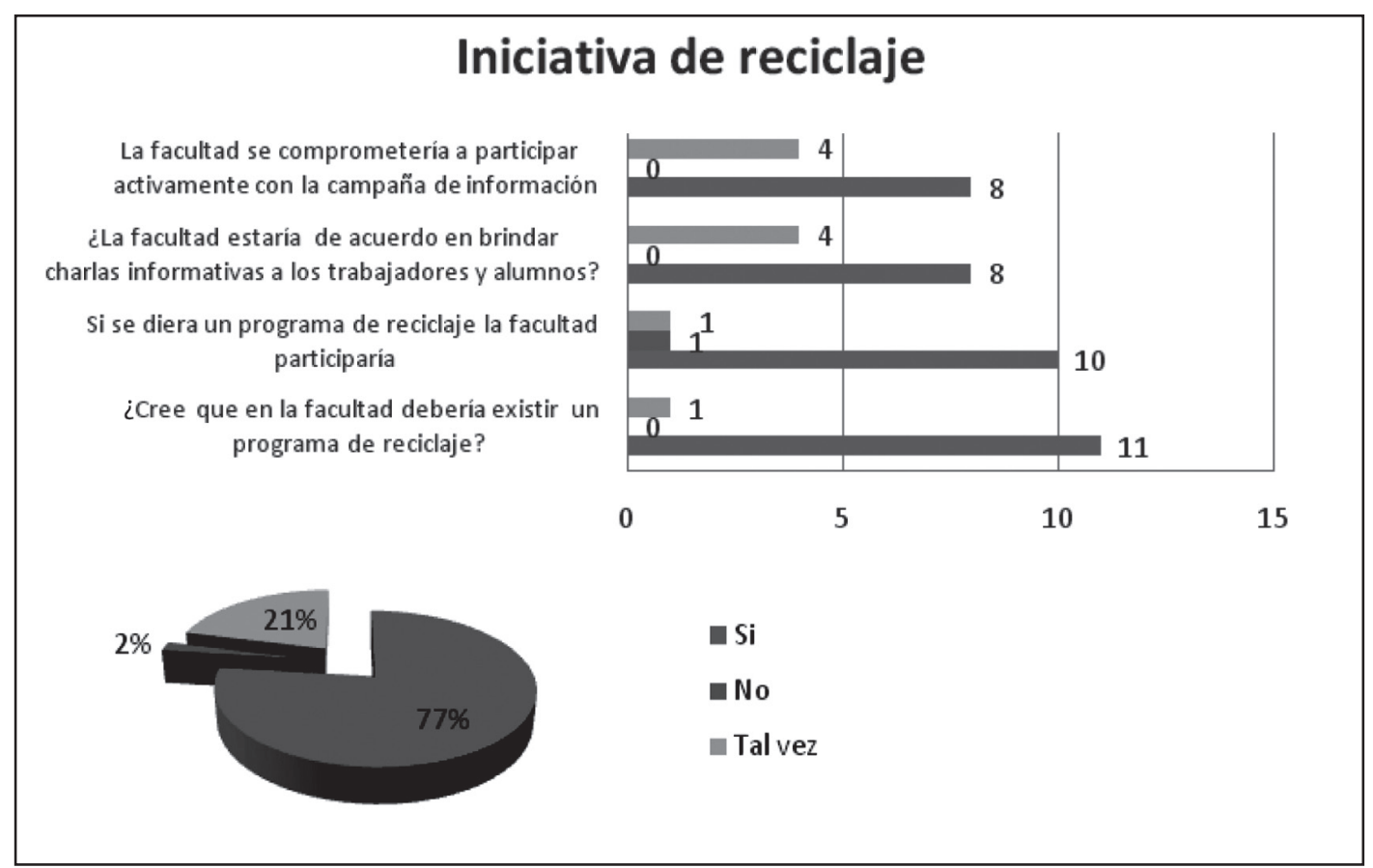

Fuente: Elaboración propia. 


\subsection{Generación de residuos sólidos}

A fin de conocer la cantidad de residuos sólidos que se emite dentro del campus universitario, se aplicaron encuestas a los jefes del personal de limpieza de las diferentes facultades (16), escuelas (60) y dependencias (12), determinándose lo siguiente:

Producción de desechos:

36 Toneladas por semana

Tipo de desechos que se produce:

7.0 Toneladas de papel por semana

3.6 Toneladas de plástico por semana

\subsection{Toneladas de vidrio por semana}

1.8 Toneladas de residuo metálico por semana

21.8 Toneladas de residuo orgánico por semana

Figura 5. Porcentaje de desechos según clasificación.

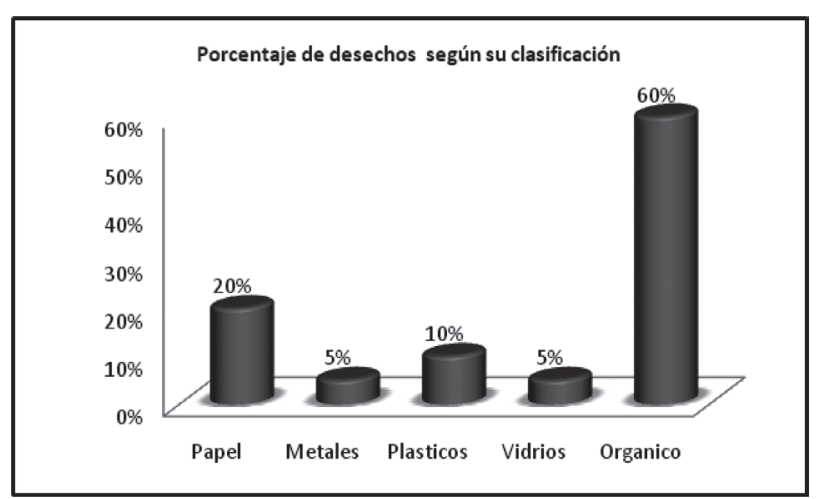

Fuente: Elaboración propia.

\subsection{Proceso de recolección}

Para conocerla realidad sobre el tratamiento de los desechos generados en los diversos ambientes del campus universitario, se realizaron entrevistas a los jefes de almacén de las facultades y dependencias universitarias, así como encuestas a los jefes de personal de limpieza de las facultades, encontrándose que el proceso de recolección se realiza en dos grandes fases (Figura 4).
Figura 6. Diagrama del proceso de recolección.

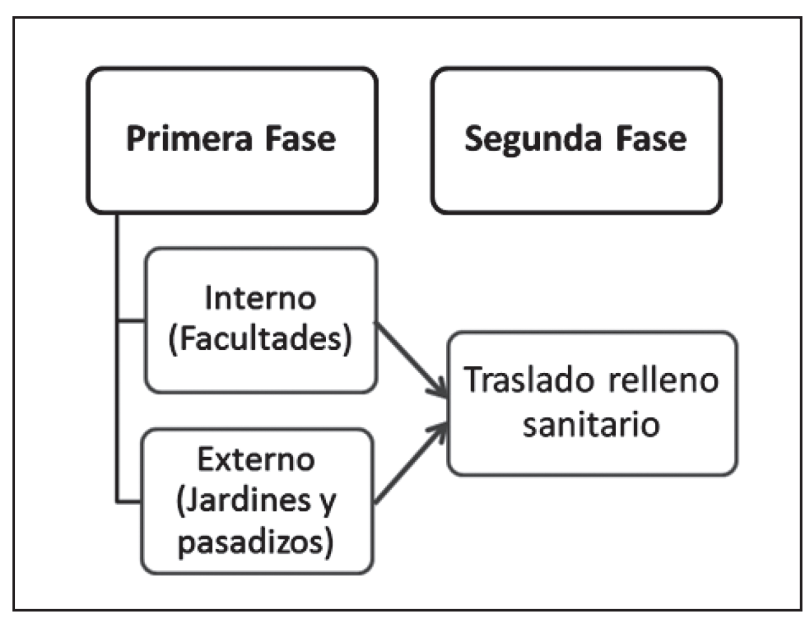

Fuente. Elaboración propia.

\subsubsection{Primera Fase}

La primera fase corresponde al traslado de los residuos de las facultades a los puntos de acopio, los cuales están distribuidos en la ciudad universitaria según muestra el Cuadro 1; a su vez este traslado se realiza en dos niveles:

El nivel interno es el que se realiza dentro de cada facultad y está a cargo del personal de limpieza. En general las facultades tienen a dos encargados de limpieza, estos trabajan usualmente en turnos de 8:00 a 16:00 horas, este régimen no se aplica en la facultad de Ciencias Administrativas que tiene más personal de servicio con tres turnos de trabajo rotativo.

El nivel externo es el que se realiza en los exteriores de las dependencias y facultades de la universidad y está a cargo de una empresa privada (SURPERLIMP SAC), la cual cuenta con 16 trabajadores y un supervisor. El servicio de barrido en todas las instalaciones se realiza de lunes a sábado en el horario de 7:30 a 15:30 horas.

Antes del traslado, en ambos niveles, no existe procedimiento de segregación de manera establecida que deba seguir el personal, sin embargo los trabajadores siguen un procedimiento interno.

\subsubsection{Segunda Fase}

La segunda fase corresponde al traslado de los residuos al relleno sanitario con la ayuda de un camión recolector a cargo de una empresa privada (Tecnologías Ecológicas Prisma S.A.C), quienes realizan tres traslados por semana; en esta etapa tampoco se realiza alguna segregación. El pago por los servicios de esta fase en el año 2012 fue de S/. 94,872.00. 
Cuadro 1. Distribución de centros de acopio dentro del campus universitario

\begin{tabular}{|c|l|c|}
\hline \multirow{4}{*}{ CENTROS DE ACOPIO } & \multicolumn{1}{|c|}{ UBICACIÓN } & CONTENEDORES \\
\cline { 2 - 3 } & Exteriores de la Facultad de Sistemas & 3 \\
\cline { 2 - 3 } & Frente a la Facultad de Ciencias Biológicas & 3 \\
\cline { 2 - 3 } & Frente a la Facultad de Mecánica de Fluidos y Letras & 3 \\
\cline { 2 - 3 } & Frente a la Facultad de Geología & 1 \\
\hline
\end{tabular}

Fuente. Elaboración propia.

Figura 7. Contenedor ubicado al frente de la facultad de Biología.

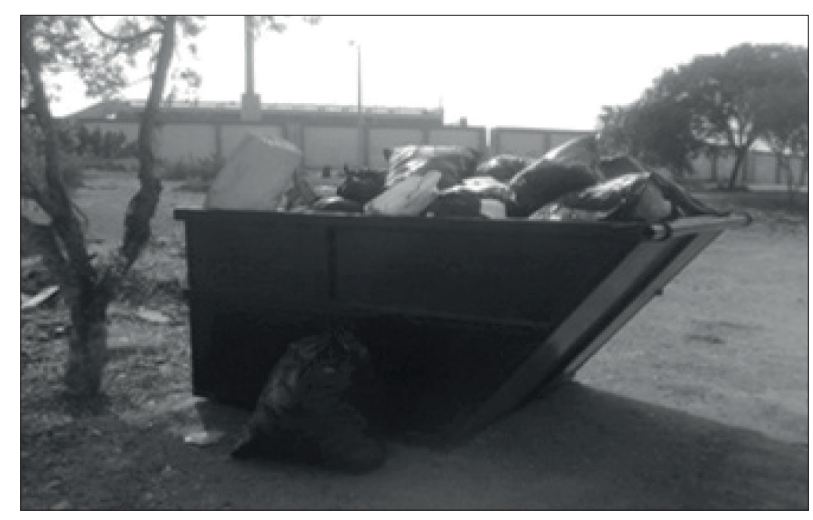

Fuente. Elaboración propia.
Figura 8. Contenedor ubicado al costado de la facultad de Sistemas.

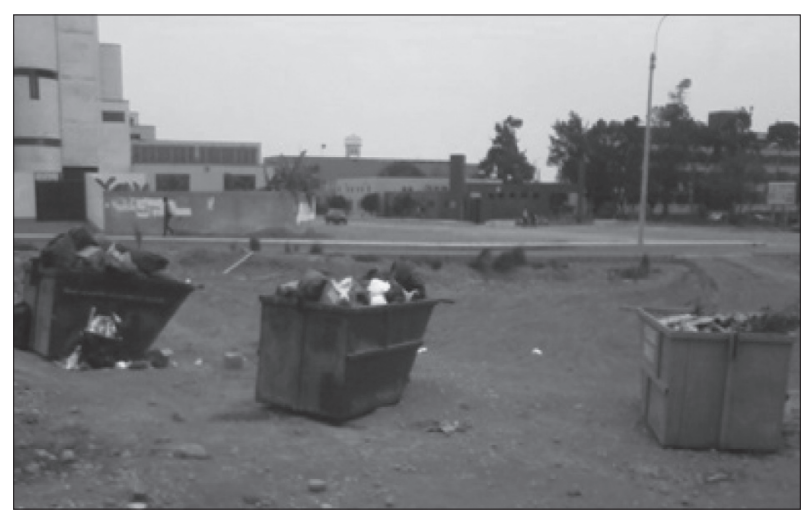

Fuente. Elaboración propia.

\subsection{Análisis interno del tratamiento de residuos sólidos}

Este análisis se ha realizado luego de la evaluación de los procesos que incluyen este servicio y en los cuales se puede determinar la estrategia adecuada para viabilizar un proceso eficiente.

\section{Cuadro 2. Matriz FODA}

\begin{tabular}{|c|c|}
\hline Fortalezas & Oportunidades \\
\hline $\begin{array}{l}\text { Existe la Oficina General de Servicios Generales, } \\
\text { Operaciones y Mantenimiento, la que se encarga } \\
\text { de la gestión de los desechos dentro de la Ciudad } \\
\text { Universitaria. } \\
\text { * En cada facultad las Oficinas de Servicios Generales y } \\
\text { Mantenimiento, gestionan los desechos. }\end{array}$ & $\begin{array}{l}\text { * Creciente iniciativa a la participación futura de un } \\
\text { programa de reciclaje por parte de la comunidad } \\
\text { universitaria. } \\
\text { * Generar una planta piloto de carácter docente. } \\
\text { * Convenios con instituciones externas que apoyan el } \\
\text { ecosistema. }\end{array}$ \\
\hline Debilidades & Amenazas \\
\hline $\begin{array}{l}\text { La comunidad universitaria carece de una cultura } \\
\text { ambiental. } \\
\text { * No se realiza capacitaciones acerca del cuidado del } \\
\text { medio ambiente. } \\
\text { * No existe una segregación de los residuos sólidos. } \\
\text { abasto para el volumen de desechos generados. } \\
\text { - Carencia de procedimiento para el proceso de } \\
\text { recolección de desechos. }\end{array}$ & $\begin{array}{l}\text { *a falta de tratamiento de los residuos generados } \\
\text { podrían generar casos de insalubridad. } \\
\text { * Posibilidad de amonestaciones de organismos } \\
\text { gubernamentales. }\end{array}$ \\
\hline
\end{tabular}

Fuente: Elaboración propia. 


\subsection{Medición de la eficiencia del tratamiento actual de los residuos sólidos}

Para esta medición se ha tomado en consideración los factores que influyen en un recojo de desechos eficiente, Cuadro 3 ysu respectiva calificación en el Cuadro 4

Cuadro 3. Factores de evaluación

\begin{tabular}{|l|c|}
\hline \multicolumn{1}{|c|}{ Factores } & Ponderación \\
\hline Capacidad de almacenamiento & 30 \\
\hline Cobertura de recolección & 20 \\
\hline $\begin{array}{l}\text { Capacitación de personal } \\
\text { involucrado }\end{array}$ & 15 \\
\hline Tecnología empleada & 15 \\
\hline Segregación eficiente & 20 \\
\hline & 100 \\
\hline
\end{tabular}

Fuente: Elaboración propia.

Cuadro 4. Criterios de calificación

\begin{tabular}{|l|c|}
\hline \multicolumn{1}{|c|}{ Criterio } & Calificación \\
\hline No es importante & 1 \\
\hline Poco importante & 2 \\
\hline Regular & 3 \\
\hline Importante & 4 \\
\hline Muy importante & 5 \\
\hline
\end{tabular}

Fuente: Elaboración propia.

Se realizaron las siguientes observaciones:

a. EI recojo de los residuos sólidos generados en la UNMSM, se realiza sólo tres veces por semana, lo cual no es suficiente y genera malestar dentro de las facultades cercanas a los contenedores de desechos por el olor y la propagación de roedores, sobre todo en meses de verano.

b. La capacidad de los contenedores existentes es insuficiente para la creciente población del campus universitario. No existe clasificación alguna de los residuos almacenados en estos contenedores.

c. El personal asignado para el recojo de residuos es insuficiente y en su mayoría no están capacitados para la separación de los residuos sólidos. d. La separación de los desechos en la mayoría de dependencias se realiza de manera informal y a pequeña escala, solo se recogen los residuos sólidos que se pueden comercializar como papel, cartón y plástico, esta separación es realizada por el personal de limpieza.

Se obtuvo el siguiente resultado para la situación actual del sistema de recojo de desechos:

Cuadro 5. Evaluación del tratamiento actual de residuos sólidos

\begin{tabular}{|l|r|r|}
\hline \multicolumn{1}{|c|}{ Factores } & Calif. & Puntaje \\
\hline $\begin{array}{l}\text { Capacidad de almacenamiento } \\
\text { adecuada }\end{array}$ & 2 & 60 \\
\hline Cobertura de recolección & 4 & 80 \\
\hline $\begin{array}{l}\text { Capacitación a personal } \\
\text { involucrado }\end{array}$ & 2 & 30 \\
\hline Tecnología empleada & 4 & 60 \\
\hline Segregación eficiente & 2 & 40 \\
\hline & $\mathbf{1 4}$ & $\mathbf{2 7 0}$ \\
\hline
\end{tabular}

Fuente: Elaboración propia.

Al comparar el puntaje del sistema actual con el puntaje de un sistema ideal eficiente (500 puntos), se aprecia que el sistema actual de recojo opera con un $54 \%$ de eficiencia. Por lo tanto, se puede indicar que el sistema tiene que mejorar.

\section{ALTERNATIVAS DE SOLUCIÓN}

Teniendo en cuenta los resultados de la evaluación del sistema actual, se plantean tres alternativas de solución a este problema:

\section{Alternativa $\mathbf{N}^{\circ}{ }^{\circ} 1$}

Servicio externo de recolección y limpieza

Se propone la conservación del sistema actual de recojo con modificaciones: control del volumen y el destinode losresiduos.

\section{Alternativa $\mathrm{N}^{\circ} \mathbf{2}$}

Implementación de servicio interno de recolección

Se aplica a la recolección de los desechos dentro de la ciudad universitaria (de manera externa, pistas y veredas, e interna, facultades y entidades). La propuesta está basada en la implementación de nuevos puntos de acopio distribuidos de manera estratégica dentro del campus universitario, nuevos 
contenedores de mayor volumen de acuerdo a la necesidad de la población universitaria, debidamente clasificados y señalizados.

\section{Alternativa N. ${ }^{\circ} 3$}

Implementación de ruta y segregación en planta procesadora

A la alternativa anterior se suma la implementación de una planta de tratamiento de residuos, que recibirá y procesará los residuos generados dentro de la ciudad universitaria con el fin de obtener beneficios de salubridad y económicos. Por otro lado, esta alternativa también tiene un fin académico, ya que servirá como una planta piloto destinada al uso y servicio de la comunidad universitaria donde puedan aplicar los conocimientos adquiridos por las distintas escuelas profesionales de la universidad.

Estas alternativas han sido evaluadas y comparadas, tomando en consideración los factores de evaluación de alternativas que se utilizan en el sector privado y estatal que se presentan en el Cuadro 6, según la calificación del Cuadro 4.

Cuadro 6. Factores de evaluación de alternativas

\begin{tabular}{|l|c|}
\hline \multicolumn{1}{|c|}{ Factores } & Ponderación \\
\hline Costos de implementación & 25 \\
\hline Cobertura del servicio & 20 \\
\hline Alcance del reciclaje & 20 \\
\hline Calidad del servicio & 18 \\
\hline Nivel de Tecnología usada & 17 \\
\hline & 100 \\
\hline
\end{tabular}

Fuente: Elaboración propia.
Del Cuadro 7 se puede señalar que la mejor alternativa es la tercera que propone la implementación de una nueva ruta de recojo de desechos así como la creación de una planta para el tratamiento de los residuos sólidos reciclables.

\section{IMPLEMENTACIÓN DE LA ALTERNATIVA PROPUESTA}

\subsection{Nueva ruta de recojo}

Esta nueva ruta de recojo se divide en dos niveles:

\subsubsection{Nivel externo}

En el nivel externo se propone una nueva ruta de recojo que comprende los exteriores de las dependencias y facultades de la ciudad universitaria, así como la construcción e implementación de ocho (08) nuevos puntos de acopio debidamente señalizados (se tuvo en consideración cantidad de desechos y distancia de transporte), cumpliendo las directivas de ecoeficiencia. Cada punto de acopio tendrá cinco (05) contenedores para el almacenamiento temporal de los residuos, diferenciados por colores según el código NTP 900.058.2005 - "Gestión ambiental", establecido por INDECOPI.

La distribución de los nuevos puntos de acopio se muestra en la Figura 7.

Se considera en la implementación un vehículo (motocarga) que transportará todos los desechos generados desde los puntos de acopio a la planta de reciclaje, la capacidad de este vehículo es de media tonelada.

Cuadro 7. Evaluación de las alternativas de solución

\begin{tabular}{|l|r|r|r|r|r|r|}
\hline & \multicolumn{2}{|c|}{ Alternativa 01 } & \multicolumn{2}{c|}{ Alternativa 02 } & \multicolumn{2}{c|}{ Alternativa 03 } \\
\hline Factores & Calif. & Puntaje & Calif. & Puntaje & \multicolumn{1}{c|}{ Calif. } & Puntaje \\
\hline Costos de implementación & 3 & 75 & 4 & 100 & 5 & 125 \\
\hline Cobertura del servicio & 3 & 60 & 5 & 100 & 4 & 80 \\
\hline Alcance del reciclaje & 3 & 60 & 3 & 60 & 5 & 100 \\
\hline Calidad del servicio & 4 & 72 & 3 & 54 & 4 & 72 \\
\hline Nivel de Tecnología usada & 3 & 51 & 3 & 51 & 4 & 68 \\
\hline & & 318 & & 365 & & \\
\hline
\end{tabular}

Fuente: Elaboración propia. 
Figura 9. Distribución de los 8 puntos de acopio.

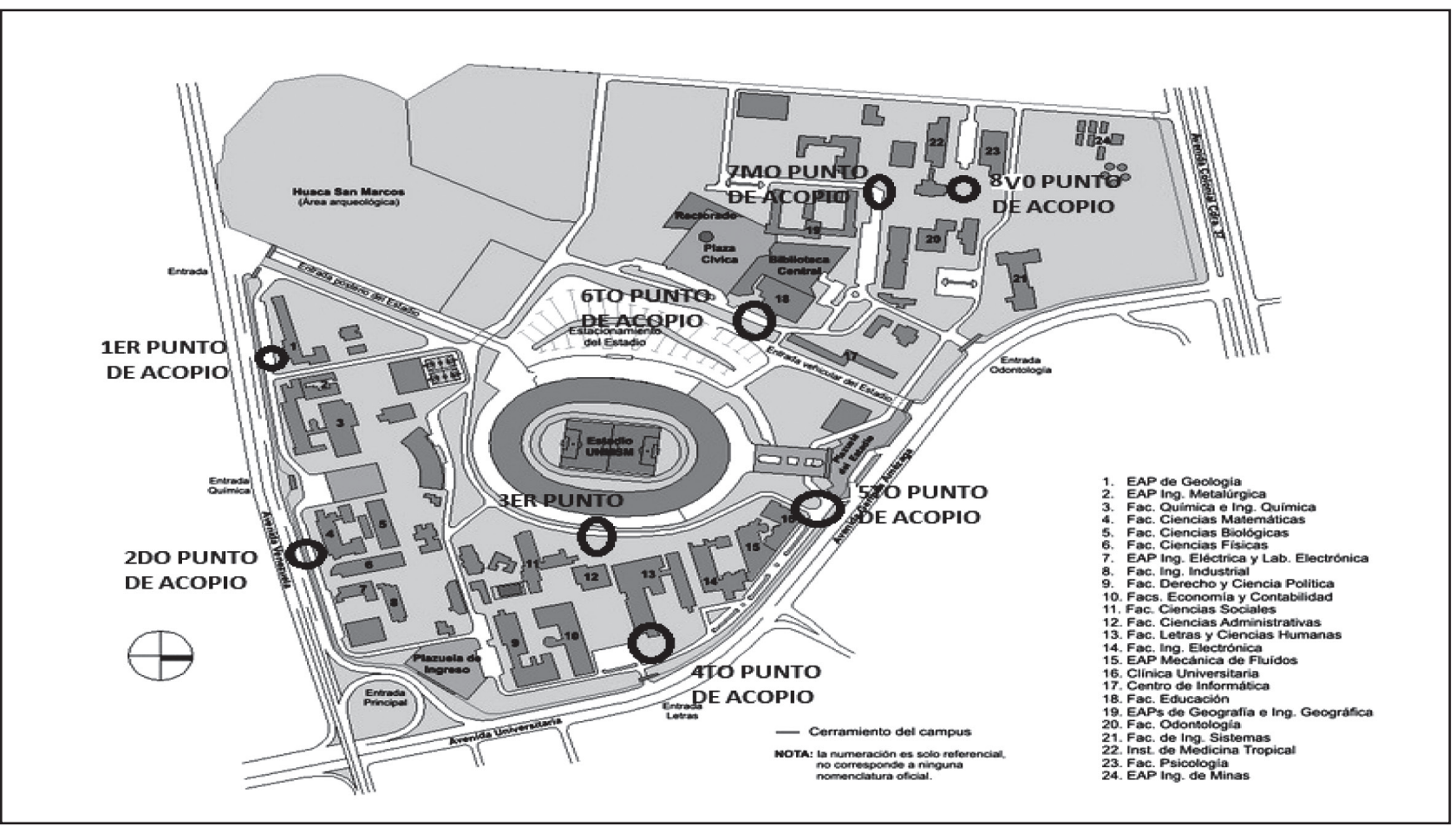

Fuente. Elaboración propia.

\subsubsection{Nivel interno}

En el nivel interno se propone implantar operaciones de segregación dentro de las facultades y dependencias de la ciudad universitaria. Cada facultad o dependencia es la encargada de su propio sistema de separación y recolección de residuos, será planificada y ejecutada por el área de mantenimiento de cada dependencia o facultad, para ello se deberá capacitar e inducir al personal involucrado asegurando mayor eficiencia. Crear cultura ambiental en la comunidad universitaria de manera que los residuos se han dejados en sus respectivos contenedores.

\subsection{Ubicación de la planta de tratamiento de residuos}

Para la elección de la ubicación de la planta se tomó en consideración los terrenos disponibles del campus universitario. Se emplea el método ponderado para las siguientes tres alternativas:
Alternativa $\mathrm{N}^{\circ}$ 1: Planta ubicada detrás de la huaca.

Alternativa $\mathbf{N} .^{\circ}$ 2: Planta ubicada al costado de maestranza.

Alternativa $\mathrm{N}^{\circ}$ 3: Planta ubicada detrás de la facultad de ingeniería de sistemas

En el Cuadro 8 se muestra los factores establecidos para la toma de decisión con sus ponderaciones. Se consideró números del 1 al 4 según cualidad, se muestra en el Cuadro 9.

El Cuadro 10 muestra que la alternativa $N .^{\circ} 2$ (mayor puntaje) es la mejor opción para la ejecución del proyecto. En la Figura 10 se muestra el terreno seleccionado de $1200 \mathrm{~m}^{2}(40 \mathrm{~m} \times 30 \mathrm{~m})$ y en la Figura 11 su ubicación dentro del campus universitario. 
Cuadro 8. Factores y ponderación

\begin{tabular}{|l|l|c|c|c|c|}
\hline & Factores & Ponderación & Alter. 01 & Alter. 02 & Alter. 03 \\
\hline 1. & Fácil acceso & $30 \%$ & 1 & 3 & 2 \\
\hline 2. & Área disponible & $25 \%$ & 4 & 4 & 3 \\
\hline 3. & Rutas habilitadas de acceso & $25 \%$ & 2 & 4 & 2 \\
\hline 4. & Suministros Básicos & $20 \%$ & 2 & 3 & 2 \\
\hline & TOTAL & $100 \%$ & & & \\
\hline
\end{tabular}

Fuente: Elaboración propia.

Cuadro 9. Puntaje

\begin{tabular}{|c|c|}
\hline Calificación & Puntaje \\
\hline Mala & 1 \\
\hline Regular & 2 \\
\hline Adecuado & 3 \\
\hline Muy bueno & 4 \\
\hline
\end{tabular}

Fuente: Elaboración propia.
Cuadro 10. Evaluación de las alternativas de ubicación

\begin{tabular}{|l|c|c|c|}
\hline \multirow{2}{*}{\multicolumn{1}{|c|}{ FACTORES }} & \multicolumn{3}{c|}{ CALIFICACIÓN } \\
\cline { 2 - 4 } & Alter. 01 & Alter. 02 & Alter. 03 \\
\hline Fácil acceso & 0.3 & 0.9 & 0.6 \\
\hline Área disponible & 1 & 1 & 0.75 \\
\hline Rutas habilitadas de acceso & 0.5 & 1 & 0.5 \\
\hline Suministros Básicos & 0.4 & 0.6 & 0.4 \\
\hline \multicolumn{1}{|c|}{ TOTAL } & $\mathbf{2 . 2}$ & $\mathbf{3 . 5}$ & $\mathbf{2 . 2 5}$ \\
\hline
\end{tabular}

Fuente: Elaboración propia.
En la Figura 12 se aprecia la distribución de planta del $1 \mathrm{er}$ piso de la planta procesadora. En el segundo

Figura 10. Terreno para el proyecto.

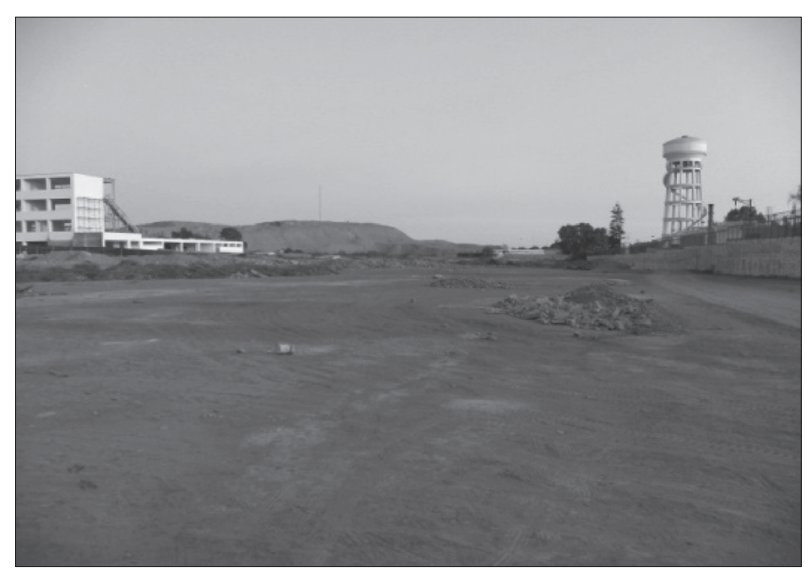

Fuente: Elaboración propia. piso sólo se construirán $78 \mathrm{~m}^{2}$, donde funcionarán las oficinas administrativas.

Figura 11. Ubicación del terreno. Biblioteca Central

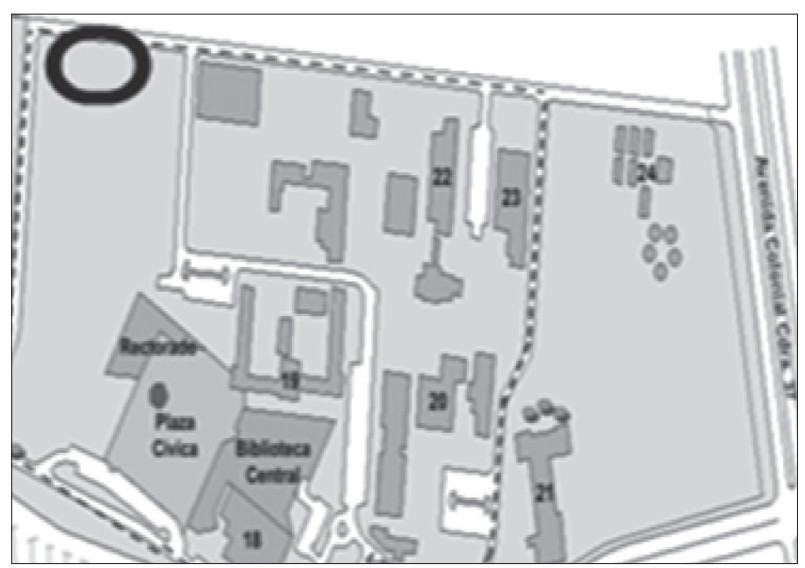

Fuente: Elaboración propia. 


\subsection{Distribución de planta}

Figura 12. Distribución de planta de la planta procesadora.

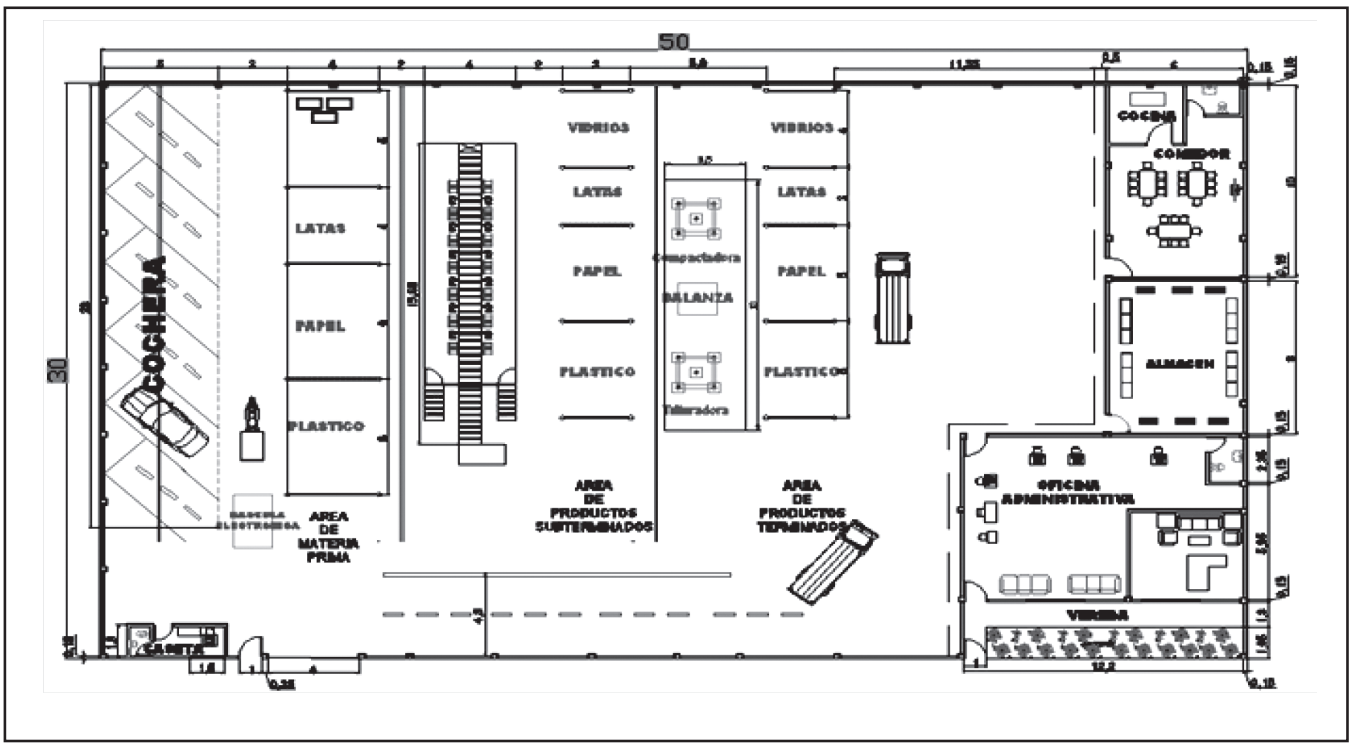

\subsection{Inversión}

La inversión total para la implementación de la planta procesadora se muestra en el Cuadro 11, para lo cual se ha considerado básicamente cuatro componentes:

\section{a. Construcción de local}

La cantidad requerida para la construcción de la planta es de 465,435.60 Nuevos Soles.

\section{b. Activos tangibles}

Dentro de la tecnología de planta se consideró las maquinarias, muebles, etc. que se utilizara para implementar la planta.

\section{c. Activos no tangibles}

Se considerará a los estudios previo ( $1 \%$ de la inversión) donde la inversión es S/. 800,016.10 (comprende la construcción de la planta y activos tangibles) y la constitución de la empresa estimada en S/. 500.00

\section{d. Capital de trabajo}

Es la cantidad requerida para que se necesitara para la puesta en marcha de la planta de reciclaje.
Cuadro 11. Inversión para la implementación depuesta en marcha de la planta procesadora

\begin{tabular}{|l|lr|}
\hline \multicolumn{2}{|c|}{ Costos de Inversión } \\
\hline Activos tangibles & S/. & $800,016.10$ \\
\hline Activos no Tangibles & S/. & $13,000.00$ \\
\hline Capital de Trabajo & S/. & $63,876.00$ \\
\hline TOTAL & S/. & $\mathbf{8 7 6 , 8 9 2 . 1 0}$ \\
\hline
\end{tabular}

Fuente. Elaboración propia.

\section{CAPACITACIÓN Y PUBLICIDAD GENERADA}

Para la implementación de la alternativa propuesta se requiere una capacitación adecuada considerando la importancia del reciclaje, el proceso deseleccióny la recolección del manejo de los residuos sólidos, así como la oportunidad de generar recursos a través de ellos.

Siendo este un tema de conservación del medio ambiente y considerándola dentro de la proyección social que debe de tener la universidad, como entidad del estado frente a la sociedad, se debe incentivar la participación de todo el personal involucrado, llámese docentes, personal administrativo, personal 
operativo, alumnado en general y personal que brinda servicios en kioskos, librerías y cafeterías; no solo en la capacitación, divulgación y ejecución del proceso, sino también en la supervisión de la correcta realización por parte de personas externas a la universidad.

En la Figura 13 se muestra el logo de difusión a utilizar en la capacitación:

Figura 13. Logo de difusión depuesta en marcha de la planta procesadora.

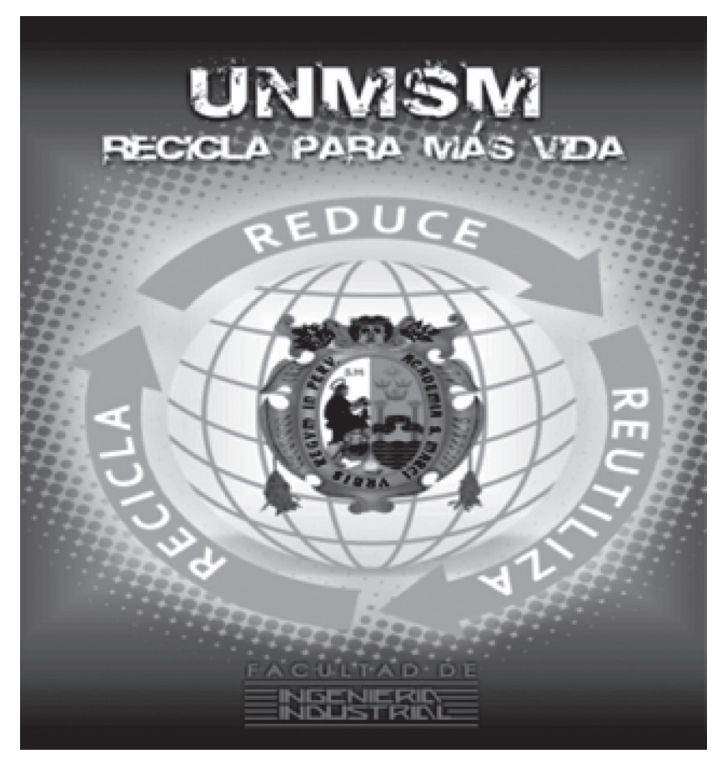

\section{CONCLUSIONES}

- La gestión del recojo de desechos dentro del campus universitario mejorará con la implementación de la planta de tratamiento de residuos.

- La planta de tratamiento de residuos también tendrá un fin académico donde los estudiantes puedan aplicar los conocimientos adquiridos en las aulas, estará ubicada en dentro del campus universitario.

- El recojo actual de la basura es deficiente

- La comunidad universitaria carece de una cultura ambiental.

\section{REFERENCIAS BIBLIOGRÁFICAS}

[1] Ecología Verde, (2009). Los beneficios de reciclar.Recuperado 08 de agosto de 2013, de http://www.ecologiaverde.com/los-beneficiosde-reciclar/

[2] Manual para Municipios Ecoeficientes, (2009). Recuperado 10 de agosto de 2013, de http:// www.minamb.gob.pe.

[3] Que es el reciclaje. Recuperado 08 de agosto de 2013, de http://www.yolimpio.com/recicla.

[4] Plan general de residuos. Recuperado 20 de setiembre de 2013, de http://www. uva.es/export/sites/default/portal/adjuntos/ documentos/1241607314333_plan_general_ residuos.pdf 